\title{
Two different approaches to the Affective Profiles Model: Median splits (variable-oriented) and cluster analysis (person- oriented)
}

Danilo Garcia, Shane MacDonald, Trevor Archer

Background: The notion of the affective system as being composed of two dimensions led Archer and colleagues to the development of the affective profiles model. The model consists of four different profiles based on combinations of individuals' experience of high/low positive and negative affect: self-fulfilling, low affective, high affective, and selfdestructive. During the past 10 years, an increasing number of studies have used this person-centered model as the backdrop for the investigation of between and within individual differences in ill-being and well-being. The most common approach to this profiling is by dividing individuals' scores of self-reported affect using the median of the population as reference for high/low splits. However, scores just-above and just-below the median might become high and low by arbitrariness, not by reality. Thus, it is plausible to criticize the validity of this variable-oriented approach. Our aim was to compare the median splits approach with a person-oriented approach, namely, cluster analysis.

Method: The participants ( $N=2,225)$ were recruited through Amazons' Mechanical Turk and asked to self-report affect using the Positive Affect Negative Affect Schedule. We compared the profiles' homogeneity and Silhouette coefficients to discern differences in homogeneity and heterogeneity between approaches. We also conducted exact cell-wise analyses matching the profiles from both approaches and matching profiles and gender to investigate profiling agreement with respect to affectivity levels and affectivity and gender. All analyses were conducted using the ROPstat software.

Results: The cluster approach (weighted average of cluster homogeneity coefficients = 0.62 , Silhouette coefficients $=0.68$ ) generated profiles with greater homogeneity and more distinctive from each other compared to the median splits approach (weighted average of cluster homogeneity coefficients $=0.75$, Silhouette coefficients $=0.59$ ). Most of the participants ( $n=1736,78.02 \%$ ) were allocated to the same profile (Rand Index $=$ .83), however, 489 (21.98\%) were allocated to different profiles depending on the approach. Both approaches allocated females and males similarly in three of the four 
profiles. Only the cluster analysis approach classified men significantly more often than chance to a self-fulfilling profile (type) and females less often than chance to this very same profile (antitype).

Conclusions: Although the question whether one approach is more appropriate than the other is still without answer, the cluster method allocated individuals to profiles that are more in accordance with the conceptual basis of the model and also to expected gender differences. More importantly, regardless of the approach, our findings suggest that the model mirrors a complex and dynamic adaptive system. 
1 Under editorial evaluation in PeerJ

2 Please do not quote without permission

3

4

5

6

16

17

\section{Two different approaches to the Affective Profiles Model:}

Median splits (variable-oriented) and cluster analysis (person-oriented)

Danilo Garcia ${ }^{1,2,3,4 *}$, Shane MacDonald $3,5,6$, Trevor Archer ${ }^{2,3}$

${ }^{1}$ Blekinge Centre of Competence, Blekinge County Council, Karlskrona, Sweden

${ }^{2}$ Department of Psychology, University of Gothenburg, Gothenburg, Sweden

${ }^{3}$ Network for Empowerment and Well-Being, Sweden

${ }^{4}$ Centre for Ethics, Law and Mental Health (CELAM), University of Gothenburg, Gothenburg,

Sweden

${ }^{5}$ Center for Health and Medical Psychology (CHAMP), Psychological Institution, Örebro University, Örebro, Sweden

${ }^{6}$ Psychological Links of Unique Strengths (PLUS), Psychological Institution, Stockholm University, Stockholm, Sweden 
18 * Correspondence concerning this article should be addressed to D. Garcia, Network for 19 Empowerment and Well-Being, Axel W. Anderssons Väg 8A, SE 37162 Lyckeby, Sweden. E20 mail: danilo.garcia@icloud.com; danilo.garcia@neuro.gu.se. 


\begin{abstract}
Background: The notion of the affective system as being composed of two dimensions led Archer and colleagues to the development of the affective profiles model. The model consists of four different profiles based on combinations of individuals' experience of high/low positive and negative affect: self-fulfilling, low affective, high affective, and self-destructive. During the past 10 years, an increasing number of studies have used this person-centered model as the backdrop for the investigation of between and within individual differences in ill-being and well-being. The most common approach to this profiling is by dividing individuals' scores of self-reported affect using the median of the population as reference for high/low splits. However, scores justabove and just-below the median might become high and low by arbitrariness, not by reality. Thus, it is plausible to criticize the validity of this variable-oriented approach. Our aim was to compare the median splits approach with a person-oriented approach, namely, cluster analysis.
\end{abstract}

Method: The participants $(N=2,225)$ were recruited through Amazons' Mechanical Turk and asked to self-report affect using the Positive Affect Negative Affect Schedule. We compared the profiles' homogeneity and Silhouette coefficients to discern differences in homogeneity and heterogeneity between approaches. We also conducted exact cell-wise analyses matching the profiles from both approaches and matching profiles and gender to investigate profiling agreement with respect to affectivity levels and affectivity and gender. All analyses were conducted using the ROPstat software.

Results: The cluster approach (weighted average of cluster homogeneity coefficients $=0.62$, Silhouette coefficients $=0.68$ ) generated profiles with greater homogeneity and more distinctive from each other compared to the median splits approach (weighted average of cluster homogeneity coefficients $=0.75$, Silhouette coefficients $=0.59)$. Most of the participants $(n=$ $1736,78.02 \%$ ) were allocated to the same profile (Rand Index $=.83)$, however, $489(21.98 \%)$ were allocated to different profiles depending on the approach. Both approaches allocated females and males similarly in three of the four profiles. Only the cluster analysis approach classified men significantly more often than chance to a self-fulfilling profile (type) and females less often than chance to this very same profile (antitype).

Conclusions: Although the question whether one approach is more appropriate than the other is still without answer, the cluster method allocated individuals to profiles that are more in accordance with the conceptual basis of the model and also to expected gender differences. More importantly, regardless of the approach, our findings suggest that the model mirrors a complex and dynamic adaptive system. 
67 Several health characteristics are associated with individuals' affectivity (Watson \& Tellegen,

1985); consequently, both positive affect and negative affect possess some degree of explanatory

value (e.g. Clark \& Watson, 1988). In this context, Wilson and colleagues (1998) indicated that there is no significant correlation between positive affect and negative affect as measured by one of the most common instruments used to self-report affect, the Positive Affect Negative Affect Schedule (Watson, Clark \& Tellegen, 1988). Moreover, each one of these dimensions (i.e., positive affect and negative affect) correlates to different personality and health attributes (Garcia, 2011; Norlander, Bood \& Archer, 2002). Individuals characterized by high levels of positive affect exhibit a greater appreciation of life, more security, self-esteem, and selfconfidence (Archer, Adolfson \& Karlsson, 2008; Costa \& McCrae, 1980). They enjoy more social relations and assertiveness and are generally described as passionate, happy, energetic, and alert (Watson \& Clark, 1984; Watson \& Pennebaker, 1989). In contrast, individuals characterized by high levels of negative affect experience greater stress, strain, anxiety, and uncertainty over a wide range of circumstances and events (Spector \& O’Connell, 1994; Watson, Pennebaker \& Folger, 1986). In other words, these two dimensions that compose the affective system are uncorrelated from each other. However, even in the case of null correlations there might still be a nonlinear dependency between these two affectivity dimensions. For instance, from a person-centered framework these two affectivity dimensions within the individual can be seen as interwoven components with whole-system properties (Bergman \& Wångby, 2014). The outlook of the individual as a whole-system unit is then best studied by analyzing patterns of information (Bergman \& Wångby, 2014). Although at a theoretical level there is a myriad of probable patterns of combinations of peoples' levels of positive and negative affect, if viewed at a global level, there should be a small number of more frequently observed patterns or "common 
90 types” (Bergman \& Wångby, 2014; Bergman \& Magnusson, 1997; see also Cloninger, Svrakic

91 \& Svrakic, 1997, who explain nonlinear dynamics in complex adaptive systems).

In this line of thinking, Archer and colleagues (e.g., Archer, Adrianson, Plancak \& Karlsson, 2007, Garcia, 2011; Norlander, Bood \& Archer, 2002, Norlander, von Schedvin \& Archer, 2005) coined the notion of the affective profiles by proposing four possible combinations using individuals' experience of high/low positive/negative affect: (1) high positive affect and low negative affect (i.e., the self-fulfilling profile), (2) low positive affect and low negative affect profile). During the last 10 years, research using the affective profiles model has distinguished individual differences in positive (i.e. well-being) and negative (i.e. ill-being) psychological and somatic health (e.g., Garcia, Rosenberg, Erlandsson \& Siddiqui, 2010, Garcia, Kerekes, Andersson Arntén \& Archer, 2012; Garcia \& Siddiqui, 2009ab; Garcia \& Moradi, 2013; Garcia \& Archer, 2012; Nima, Rosenberg, Archer \& Garcia, 2013; Jimmefors, Garcia, Roosenberg, Mousavi, Adrianson \& Archer, 2014). Particularly, individuals with a self-destructive profile, 105 compared to individuals with a self-fulfilling profile, experience lower subjective and psychological well-being, along with lower levels of energy, dispositional optimism, and higher levels of somatic stress, pessimism, non-constructive perfectionism, depression and anxiety, maladaptive coping, stress at the work-place, external locus of control, and impulsiveness (see among others Archer, Adrianson, Plancak \& Karlsson, 2007, Bood, Archer \& Norlander, 2004; Garcia, 2012; Garcia, Nima \& Kjell, 2014; Karlsson \& Archer, 2007; Palomo, Kostrzewa, Beninger \& Archer, 2007, Palomo, Beninger, Kostrzewa \& Archer,2008; Schütz, Archer \& 112 Garcia, 2013; Schütz, Garcia \& Archer, 2014, Schutz, Sailerm Nima, Rosenberg, Andersson 
113 Arntén, Archer \& Garcia, 2014). The most important differences, however, are discerned when

114 individuals that are similar in one affect dimension but differ in the other dimension are

115 compared to each other (Garcia, 2011). Individuals with a low affective profile (low positive

116 affect, low negative affect), for example, report to be more satisfied with their life compared to

117 individuals with a self-destructive profile (low positive affect, high negative affect). Hence,

118 suggesting that high levels of life satisfaction are associated to decreases in negative affect when

119 positive affect is low. In essence, the affective profiles model offers a nuanced representation of

120 the composition of the affectivity system - a diametrically different representation than the

121 notion of treating these two dimensions simply as two separate variables or summarizing them to

122 create one mean value (Garcia, 2011, 2012). See Figure 1 for a compilation of findings from the

123 last 10 years of research conducted by Archer, Garcia, and colleagues showing individual

124 differences and similarities using the affective profiles model.

\section{$\underline{\text { Figure } 1 \text { should be here }}$}

The most common approach to the categorization of individuals in four different affective profiles is by means of median splits. Basically, individuals' self-reported scores on positive and negative affect are divided into high and low in reference to the median (Norlander, Bood \& Archer, 2002). The individuals high and low scores are then combined into the four profiles. However, since median splits distort the meaning of high and low, it is plausible to criticize the validity of this approach to create the affective profiles-scores just-above and just-below the median become high and low by arbitrariness, not by reality (Schütz, Archer \& Garcia, 2013). That is, the median splits method is variable-oriented because it categorizes individuals in different affective profiles based on the variable's cut-off scores. A variable-oriented approach is, for instance, characterized for its focus on differences between individuals without 
136 considering the existence of sub-populations (Lundh, 2015). In this regard is plausible to suggest

137 that because the affective profiles model is, at least in theory, person-centered, it should be 138 operationalized using an approach that focuses on internal patterns, rather than individual 139 differences (cf. Lundh, 2015). Recently, MacDonald and Kormi-Nouri (2013) used person-oriented research approaches

to cluster individuals depending on their self-reported affectivity and found that the four profiles emerged as originally modeled by Archer and as operationalized using the median splits approach. However, although apparently similar, we argue that these two approaches are still different in their research focus with respect to two contrasts: (a) variable versus pattern focused and (b) individual versus population focused (cf. Lundh, 2015). The median splits approach focuses on variables and their cut-off values in populations, thus it is a top-down procedure. A bottom-up procedure, in contrast, is the hierarchical cluster analysis, which starts by sequentially joining the most similar participants on variables of interest (e.g., positive affect and negative affect) to form groups (i.e., pattern and individual focused). A follow up relocation procedure may then use K-means cluster analysis to ensure people are assigned to a profile most similar to theirs (see MacDonald \& Kormi-Nouri 2013; Kormi-Nouri, MacDonald, Farahani, Trost \& Shokri, 2015). In this respect cluster analytic methods are data-driven and create profiles that are relative to each other. Data-driven methods, compared to median splits, come closer to modeling the dynamic nature of within and between group variability of individual patterns of affectivity, while the median splits procedure is static in nature-equally sized groups are pre-determined because each one of the two variables is divided in high and low using the median.

We argue further that, depending on how profiles are made (i.e., median splits vs. cluster) the model has the potential to discern differences not found before. On average, for example, 
159

160

161

162

163

women recall experiencing negative affect to a larger extent compared to men, while on average men recall experiencing positive affect to a larger extent compared to women (e.g., Crawford \& Henry, 2004; see also Schütz, 2015). Despite this fact suggesting clear general differences in affectivity between men and women, past research using the median splits has not found interaction effects between the type of profile and the person's gender on well-being and illbeing (see Garcia, 2011). While it is plausible to suggest that the differences in affectivity between profiles overrule possible gender differences (Garcia \& Siddiqui, 2009a; Garcia, 2011), it might be so that this lack of findings depends on the choice of method to create the profiles. Indeed, in contrast to the variable-oriented method (i.e., median splits), the person-oriented method (i.e., cluster analysis) has as a primary criterion that a sample is analyzed assuming it is drawn from more than one population (von Eye \& Bogat, 2006), for example, males and females.

In sum, the aim of this paper is to compare the most often used variable-oriented median splits approach with the person-oriented cluster analysis approach when categorizing individuals into any of the four affective profiles of the model. As a first step we compared the homogeneity within the profiles created with the two different approaches and also whether the profiles created with each approach were distinct from each (i.e., heterogeneity between profiles). This was important because, according to the model, people allocated to a specific profile are expected to be similar to each other and distinct to those allocated to any of the other profiles. As a second step, we compared the two procedures to see how they agreed upon classifying people with respect to their affectivity levels. As a third and final step, we compared how males and females were allocated depending on the approach used to create the profiles.

\section{Method}


182

183

184

185

186

187

188

189

190

\section{Ethical statement}

After consulting with the Network for Empowerment and Well-Being's Review Board we arrived at the conclusion that the design of the present study (e.g., all participants' data were anonymous and will not be used for commercial or other non-scientific purposes) required only informed consent from the participants.

\section{Participants and procedure}

The participants $(N=2,225$, age mean $=31.79, s d .=15.58,1160$ males and 1065 females $)$ were $\begin{array}{llll}\text { recruited } & \text { through } & \text { Amazons' } & \text { Mechanical }\end{array}$ https://www.mturk.com/mturk/welcome). MTurk allows data collectors to recruit participants (workers) online for completing different tasks in exchange for wages. This method of data collection online has become more common during recent years and it is an empirically tested tool for conducting research in the social sciences (see Buhrmester, Kwang \& Gosling, 2011). Participants were recruited by the criteria of being a US-resident and the ability to read and write fluently in English. Participants were paid a wage of .50 cents (US-dollars) for completing the task and informed that the study was confidential and voluntary. The participants were presented with a battery of self-reports comprising the affectivity measure as well as questions pertaining to age and gender.

\section{Instrument}

Positive Affect Negative Affect Schedule (Watson, Clark \& Tellegen, 1988). Participants are instructed to rate to what extent they have experienced 20 different feelings or emotions (10 positive, such as, strong, proud, interested, and 10 negative, such as, afraid, ashamed, nervous) 
205 during the last weeks, using a 5-point Likert scale $(1=$ very slightly, $5=$ extremely $)$. We 206 averaged the individual items to derive participants' scores in each scale, that is, positive affect 207 and negative affect. Cronbach's $\alpha^{1}$ in the present study were .90 for positive affect and .88 for 208 negative affect.

209

210

211

212

213

214

215

216

217

218

219

220

221

222

223

224

225

\section{Statistical treatment}

At a general level the distribution of the positive affect scores are approximately normal (skewness $=-.18$, kurtosis $=-.30)$. The negative affect scores are heavily skewed on the right $($ skewness $=1.12$, kurtosis $=.98)$. This comes primarily from the fact that within the value range of negative affect (1-5) the median (1.70) is very close to the minimum (1). See Figure 2 for the distribution of positive and negative affect and Figures 3ab for the mean in both affectivity dimensions for each of the profiles created with the median splits and cluster approaches.

\section{Figure 2 should be here}

Figure $3 \mathrm{ab}$ should be here

Median splits. Participants' positive affect and negative affect scores were divided into high and low as the original method used in past studies (cut-off points in the present study: low positive affect $=3.00$ or less; high positive affect $=3.10$ or above; low negative affect $=1.60$ or less; and high negative affect $=1.70$ or above). The median splits method resulted in 641 individuals with a self-fulfilling profile (351 males, 290 females), 441 individuals with a low affective profile (235 males, 206 females), 529 individuals with a high affective profile (283 males, 246 females), and 614 individuals with a self-destructive profile (291 males, 323 females). This statistical procedure was conducted in SPSS version 22.

${ }_{1} \alpha=\frac{K}{K-1}\left(1-\frac{\sum_{i=1}^{K} \sigma_{Y_{i}}^{2}}{\sigma_{X}^{2}}\right)$, retrieved from https://en.wikipedia.org/wiki/Cronbach\%27s_alpha 
227

228

four groups. K-means cluster analysis used the starting points from this analysis to ensure that people ended up in a group most similar to their affective profile. The cluster analysis resulted in 781 individuals with a self-fulfilling profile (431 males, 350 females), 640 individuals with a low affective profile (336 males, 304 females), 459 individuals with a high affective profile (251 males, 208 females), and 345 individuals with a self-destructive profile (142 males, 203 females). This and all analyses reported under the Results section were conducted using the ROPstat software (Vargha, Torma \& Bergman, 2015; http://www.ropstat.com).

\section{Results}

\section{Homogeneity within and heterogeneity between profiles}

See Table 1 for the composition of median splits and cluster profiles. Both approaches had only one group, the self-destructive profile, that contained individuals who were dissimilar to the extent their homogeneity coefficient ${ }^{2}$ value exceeded 1 (see Bergman, Magnusson, \& El-Khouri 2003, who suggest that a homogeneity coefficient should ideally not exceed 1 for a homogenous grouping). On basis of the model, it is expected that individuals within each profile are similar to each other (i.e., homogeneity) and that profiles are distinctive from each other (i.e., heterogeneity). Hence, we also computed a weighted average of cluster homogeneity coefficients of the profiles derived using the median splits (weighted average of cluster homogeneity coefficient $=0.75)$ and cluster approaches (weighted average of cluster homogeneity coefficient $=$ 0.62). In addition, we also report here the Silhouette coefficient ${ }^{3}$, which is an adequacy measure

${ }^{2}$ The homogeneity coefficient of a cluster is the average of the pairwise differences of cases belonging to this cluster (A. Vargha, personal communication, October 8, 2015).

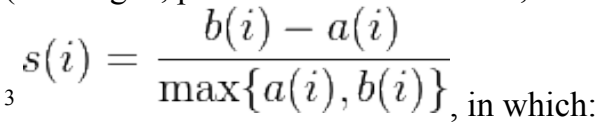

$S=$ silhouette

$i=$ each single data point

$\mathrm{a}(i)=$ the average dissimilarity of $i$ with all other data within the same cluster. That is, a $(i)$ can be interpreted as how 
246

247

248

249

250

251

252

253

254

255

256

257

258

259

260

261

262

263

264

265

266

that takes into account the participants who lie within their clusters and also the ones who are merely somewhere in between clusters (Rousseeuw, 1987). A Silhouette coefficient closer to 1 might indicate that the groups are more distinct from each other (Bergman, Magnusson \& ElKhouri, 2003). In the present sample, the cluster approach seems to generate more heterogeneous groups (Silhouette Coefficient $=0.68$ ) than those profiles created using the median splits approach (Silhouette Coefficient $=0.59$ ). Nevertheless, because the Silhouette Coefficient takes into account both the homogeneity of the clusters and the level of separation of the different clusters, the most accurate proof of heterogeneity between profiles is the differences between approaches in their weighted average of cluster homogeneity coefficient. One way or another, the cluster approach seems to have created profiles with greater homogeneity within the groups and also profiles that were more distinctive between each other. One important observation is that people is allocated differently depending on the approach. For example, the percentage of people being allocated in the self-destructive profile using the cluster method were $16 \%$, while $27 \%$ were allocated in this same profile using the median splits method.

\section{Table 1 should be here}

\section{Classification by affectivity levels between approaches}

Next, we compared the two procedures to see how they agreed upon classifying people with respect to their affectivity levels using an exact cell-wise analysis. The number of people allocated in profiles formed using median splits was crossed with the number of people in profiles resulting from cluster analysis. The aim with this base model was to create a reference

well $i$ is assigned to its cluster (the smaller the value, the better the assignment). This allow us to define the average dissimilarity of point $i$ to a cluster $\mathrm{c}$ as the average of the distance from $i$ to points in $\mathrm{c}$.

$\mathrm{b}(i)=$ the lowest average dissimilarity of $i$ to any other cluster, of which $i$ is not a member. The cluster with this lowest average dissimilarity is said to be the "neighboring cluster" of $i$ because it is the next best fit cluster for point $i$ (Rousseeuw, 1987). 
267 (i.e., an estimated expected cell frequency) to which the observed cell frequency is compared 268 against (see von Eye, Bogat \& Rhodes, 2006). In short, if a specific cell contains more cases than expected under this base model, this cell indicates a relationship that exists only in this particular sector of the cross-classification, that is, it constitutes a type. If a cell, in contrast contains fewer cases than expected under the base model, this cell also indicates a local relationship, that is, it constitutes an antitype (see also Bergman \& El-Khouri, 1987). As shown in Table 2, there is general agreement between approaches when allocating people to specific affective profiles_-all cells that correspond to the same profiles indicate types. However, there were four sizable discrepancies between the approaches. Firstly, 199 individuals who were classified as having a self-destructive profile using the median splits procedure were allocated to a low affective profile when the cluster analysis approach was used. Secondly, 140 individuals who were allocated to a high affective profile using the median splits procedure were allocated to a self-fulfilling profile when the cluster analysis was used. The third discrepancy was that 40 individuals who were allocated to a high affective profile using the median splits procedure were allocated to a selfdestructive profile when the cluster analysis approach was used. The fourth and final difference was that 110 individuals who were allocated to a self-destructive profile using the median splits procedure were allocated to a high affective profile when cluster analysis was used. In sum, most of the participants $(n=1736,78.02 \%)$ were allocated to the same profile regardless of the approach being used to create the affective profiles, but 489 participants $(21.98 \%)$ were allocated to different profiles depending on the approach. The Rand Index ${ }^{4}$, a global measure for the

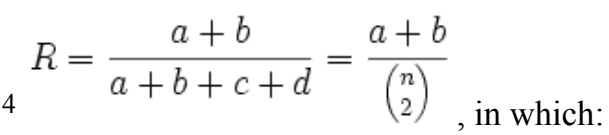

$\mathrm{a}=$ the number of pairs of elements in $\mathrm{S}$ that are in the same set in $\mathrm{X}$ and in the same set in $\mathrm{Y}$,

$\mathrm{b}=$ the number of pairs of elements in $\mathrm{S}$ that are in different sets in $\mathrm{X}$ and in different sets in $\mathrm{Y}$,

$\mathrm{c}=$ the number of pairs of elements in $\mathrm{S}$ that are in the same set in $\mathrm{X}$ and in different sets in $\mathrm{Y}$, and

$\mathrm{d}=$ the number of pairs of elements in $\mathrm{S}$ that are in different sets in $\mathrm{X}$ and in the same set in $\mathrm{Y}$.

Retrieved from https://en.m.wikipedia.org/wiki/Rand_index 
287

288

289

290

291

292

293

294

295

296

297

298

299

300

301

302

303

304

305

306

307

308

309

overall similarity of the profiling conducted by the two approaches, was .83. The Rand Index computes a similarity measure between the two profiling approaches by considering all pairs of samples and counting pairs that are assigned in the same or different profiles. The Rand Index is ensured to have a value close to 0 for random labeling independently of the number of profiles and exactly 1 when the profiling is identical. Hence, there is a large agreement between approaches.

\section{Table 2 should be here}

\section{Gender and the affective profiles}

In a third step we examined the idea of gender having an effect on profile membership. Here, the number of males and females was crossed with the number of people in profiles resulting from each of the approaches (see Table 3). The median splits and cluster analysis approaches both allocated females to a self-destructive profile more often than chance (i.e., type) and males less often than chance to this very same profile (i.e., antitype). For the high affective and the low affective profiles, both approaches allocated males and females as expected. Nevertheless, cluster analysis differed from median splits by allocating men significantly more often than chance to a self-fulfilling profile (type) and females less often than chance to a self-fulfilling profile (antitype), see Table 3. Nevertheless, the proportions of males and females allocated in the different profiles seem, on visual inspection, relatively similar for both approaches (see percentages in Table 3). The greatest discrepancies between approaches in gender distributions were found in the self-destructive profile. Specifically, in the self-destructive profile created using the median splits method the proportions within the profile were: $47.40 \%$ males and $52.60 \%$ females; while the proportions were: $41.20 \%$ males and $58.80 \%$ within the selfdestructive group created using the cluster method. 
Table 3 should be here

\section{Discussion}

The present study set out to compare two approaches (median splits vs. cluster analysis) to making profiles as derived by the notion of the affectivity system as composed of two dimension: positive affect and negative affect. In both approaches one and the same profile showed lower homogeneity, namely, the self-destructive. There were, however, three main differences: (1) both the homogeneity within profiles and the heterogeneity between profiles were significantly larger for those profiles created with the cluster method, (2) although most of the participants $(n=1736,78.02 \%)$ were allocated to the same profile regardless of the approach and a large level of agreement between approaches, a total of 489 participants $(21.98 \%)$ were allocated to different profiles, (3) and while both methods allocated males and females similarly across three of the four profiles, the methods differed in the way males and females were classified within the self-fulfilling profile. We suggest that these three differences mirror that the median splits method derives profiles focusing on variables, while the cluster method has a pattern focus that assumes the existence of data clusters, which may or may not correspond to any real subpopulations such as males and females.

According to the model (Archer, Adolfsson \& Karlsson, 2008; Norlander, Bood \& Archer, 2002; Garcia, 2011), the notion of the affectivity system as composed by two independent dimensions suggests four profiles comprising individuals who have different levels of affectivity between the profiles (i.e., heterogeneity), but have similar levels of affectivity within the profiles (i.e., homogeneity). The cluster approach generated profiles of individuals who were both more similar within (i.e., homogeneous) and more distinct from each other (i.e., heterogeneous), thus, showing that this approach is more in concordance to the theoretical basis 
333 of the affective profiles model (cf. Keren \& Schul, 2009). However, it is plausible to question

334 why both approaches show that individuals within the self-destructive profile are dissimilar from

335

336

337

338

339

340

341

342

343

344

345

346

347

348

349

350

351

352

353

354

355

each other. Importantly, low levels of positive affect and high levels of negative affect do not only characterize the self-destructive profile; this affectivity combination is also a good measure of depression (Clark \& Watson, 1991). Individuals struggling with depression have, indeed, been found to be part of a rather heterogeneous group (Goldberg, 2011). For example, although clustered together, depression patients may show opposite symptoms, such as, psychomotor retardation, hypersomnia and weight gaining in some cases, while agitation, bad sleep, and weight loss in another cases (Lux \& Kendler, 2010). In other words, both approaches seem to mirror the heterogeneity, rather than the homogeneity, within a group of individuals who experience low levels of positive affect and high levels of negative affect (i.e., the selfdestructive profile). Nevertheless, this might also imply that a four-profiles solution is not the best fit for the model.

Interestingly, 309 individuals who were allocated to the self-destructive profile using the median splits method were allocated to either the low affective $(n=199)$ or the high affective profile $(n=110)$ when the cluster method was used. Moreover, 180 individuals who were allocated to the high affective profile using the median splits method were allocated to either the self-fulfilling $(n=140)$ or the self-destructive profile $(n=40)$ when the cluster method was used. All these "moving" individuals $(n=389)$ constitute $21.98 \%$ of the total population in the present study. This "movement" might suggest that individuals who are at the very end of being high or low in relation to the median in any of the affectivity dimensions tip over when the cluster method is used. For example, the 199 individuals who "moved" from the self-destructive profile (i.e., low positive affect/high negative affect) to the low affective profile (low positive affect/low 
negative affect) are individuals who certainly are low in positive affect; but that are probably closer to the median in negative affect. In contrast, the 110 individuals who "moved" from the self-destructive profile (i.e., low positive affect/high negative affect) to the high affective profile (i.e., high positive affect/high negative affect) are individuals who certainly are high in negative affect; but are probably far way from the median in positive affect. This is, for instance, in line with our finding suggesting that the self-destructive group was the less homogeneous across both approaches. Nevertheless, most of the participants $(n=1736,78.02 \%)$ were allocated to the same profile regardless of the approach being used. We suggest that this agreement in four possible affectivity combinations reflects the affective profiles model as being conceptually personoriented. At the very least, it shows that it might be reasonable to suggest four "common types" derived of the combination of high/low positive and negative affectivity levels.

Also in this line, both methods allocated males and females similarly across three of the four profiles. Specifically, both approaches allocated females and males neither higher nor lower than expected in both the low affective and high affective profiles. In addition, both approaches allocated females to a self-destructive profile more often than chance (i.e., type) and males less often than chance to this very same profile (i.e., antitype). This specific finding across the selfdestructive profiles is in accordance to differences in affectivity between males and females (for a review see Schütz, 2015). Consequentially, this pattern also implies that the opposite should be expected, that is, with respect to the gender distribution within the self-fulfilling profile. However, only when the cluster method was applied, were males more often than expected allocated to the self-fulfilling profile (i.e., type) and females were less often than expected allocated to the self-fulfilling profile (i.e., antitype). In other words, in contrast to the median 
378 splits method, the cluster method seems to allocate individuals in profiles that mirror gender

379 differences found in the current literature (e.g., Schütz, 2015).

Nonetheless, the proportions of males and females within each profile were rather similar

381

382

383

between approaches. Remarkably, the differences in proportions were largest for the selfdestructive profile $(41.20 \%$ males and $58.80 \%$ females using the cluster method, $47.40 \%$ males and $52.60 \%$ females using the median split method) and not for the self-fulfilling profile- the only profile in which the approaches differed in the gender-pattern detailed above. Moreover, the 309 individuals who were allocated to the self-destructive profile using the median splits method, and that were allocated to either the low affective or the high affective profile when the cluster method was used, do not seem to have altered the proportions of males and females in the low affective and high affective profiles created with the cluster method. Certainly, the literature suggests that, compared to males, females have a tendency to experience high affectivity in both dimensions (Diener, Colvin, Pavot \& Allman, 1991; Diener, Sandvik \& Pavot, 1991; Garcia \& Erlandsson, 2011; Schimmack \& Diener, 1997). Still, 21.98\% of the population in the present study was allocated differently depending of the approach. We suggest that, besides gender, other variables of interest in future studies might be ethnicity, religious affiliation, and motivation. After all, these shape the emotions people want to feel—that is, their "ideal affect" (Scollon, Howard, Caldwell \& Ito, 2009; Tsai, Knutson, \& Fung, 2006; Tsai, Miao, \& Seppala, 2007; Tsai, Miao, Seppala, Fung, \& Yeung, 2007; Cloninger \& Garcia, 2015).

\section{Limitations and further suggestions}

Besides the limitations presented by a cross-sectional design (e.g., the inability to suggest in which direction participants "move" or are allocated from one profile to another depending on the approach), it is reasonable to discuss the data collection method used here (i.e., through 
401

402

403

404

405

406

407

408

409

410

411

412

413

414

415

416

417

418

419

420

421

422

423

MTurk). Some aspects related to this method might influence the validity of the results, such as, workers' attention levels, cross-talk between participants, and the fact that participants get remuneration for their answers (Buhrmester, Kwang \& Gosling, 2011). Nevertheless, a large quantity of studies show that data on psychological measures collected through MTurk meets academic standards, is demographically diverse, and also that health measures show satisfactory internal as well as test-retest reliability (Buhrmester, Kwang \& Gosling, 2011; Horton, Rand \& Zeckhauser, 2011; Shapiro, Chandler \& Mueller, 2013; Paolacci, Chandler \& Ipeirotis, 2010). In addition, the amount of payment does not seem to affect data quality; remuneration is usually small, and workers report being intrinsically motivated (e.g., participate for enjoyment) (Buhrmester, Kwang \& Gosling, 2011).

In another more important matter, the choice of approach (i.e., median splits vs. cluster) to categorize individuals in different affective profiles might depend of the distribution of the data at hand. For instance, in the present sample it seems to be evident that the median splits method does not yield naturally separable four profiles because it cuts the whole sample in cutoff points where cases are closest to each other. Due to this, cases being very close to each other may be sorted into different profiles. In addition, albeit we were interested into test the fourprofile solution suggested by Archer, even the four-cluster structure created with the cluster analysis does not seem to be a natural good solution. From a theoretical point of view, future studies might strive to find the best structure of cluster analysis and compare this to the four profiles originally suggested by Archer and colleagues. Another solution to this data-distribution problem would be to use an amalgamation of the methods. If the data have a symmetric and unimodal distribution in a dimension, it is reasonable to use median splits in that dimension. If the data has a bimodal distribution that can be well separated into two clusters in the other 
424 dimension, it is reasonable to use clustering in that dimension. In other words, the choice 425 between median splits and clustering is probable best though as dimension-wise data dependent. 426 Yet, another solution would be to create three categories with two cut-off points (e.g., with quartiles 1 and 3): one category in the middle and two on the tails.

Furthermore, future studies need to assess empirical differences in, for example, health measures between profiles created with the different approaches. Future studies should also compare the profiles created with different approaches using person-oriented techniques. In the present study, for example, we used exact cell-wise analyses to investigate if gender explained the allocation of individuals to different profiles. Although the same can be done using education level, ethnicity, and religious affiliation, and other variables of interest; there is an increasing amount of person-centered methods that can be used as detailed in recent literature (see among others Bergman \& Lundh, 2015; Valsiner, 2015; Lundh, 2015; Molenaar, 2015; Loursen, 2015; Asendorpf, 2015; von Eye \& Wiederman, 2015; Aunola, Tolvanen, Kiuru, Kaila, Mullola \& Nurmi, 2015; Vargha, Torma \& Bergman, 2015; Baker, 2015).

\section{Concluding remarks}

Our results suggest that the cluster method allocates individuals to profiles that are more in accordance with the conceptual basis of the model and also to expected gender differences. The question whether one approach is more appropriate than the other is still without answer, but the present study is only a first step in the development of the affective profiles model beyond the past 10 years of research. More importantly, regardless of the approach, the model of the affective system proposed by Archer and colleagues at the beginning of this century, actually mirrors a complex adaptive system. In other words, it is an affective system that is dynamic both between and within individuals and presents a probabilistic and exponentially complex reality. 
447 "Flowers are restful to look at. They have neither emotions nor conflicts."

448 Sigmund Freud

449 Acknowledgements

450 Firs of all we would like to express our gratitude to both reviewers, Professor Andras Vargha and

451 Professor Jingyi Jessica Li, for their comments and suggestions, which helped us to greatly

452 improve the original manuscript. We would like to thank Sophia Isabella Garcia Rosenberg and 453 Linnéa Mercedes Garcia Rosenberg for the inspiration to Figure 3a ("Joy") and 3b ("Sadness").

454 Professor Andras Vargha's suggestion for Figure 2 is also most appreciated as well as his help 455 providing the ROPstat software.

456

457

458

459

460

461

462

463

464

465

466

467

468

469

\section{References}

Adrianson L, Ancok, D., Ramdhani, N., \& Archer T (2013) Cultural influences upon health, affect, self-esteem and impulsiveness: an Indonesian-Swedish comparison. International Journal of Research Studies of Psychology, 2, 25-44. DOI: 10.5861/ijrsp.2013.228.

Archer T, Adolfsson B, Karlsson E (2008) Affective personality as cognitive-emotional presymptom profiles regulatory for self-reported health predispositions. Neurotoxicity Research, 14, 21-44. DOI 10.1007/BF03033573.

Archer T, Adrianson L, Plancak A, Karlsson E (2007) Influence of affective personality on cognitive-mediated emotional processing: need for empowerment. European Journal of Psychiatry, 21, 21-44. DOI 10.4321/S0213-61632007000400002.

Asendorpf, J. B. (2015). Person-oriented approaches whithin multi-level perspective. Journal of Person-Oriented Research, 1 (1-2), 48-55. DOI: 10.17505/jpor.2015.06. 
Aunola, K., Tolvanen, A., Kiuru, N., Kaila, K., Mullola, S., \& Nurmi, J-E. (2015). A personoriented approach to diary data. Children's temperamental negative emotionality increases susceptibility to emotion transmission in father-child dyads. Journal of PersonOriented Research, 1 (1-2), 72-86. DOI: 10.17505/jpor.2015.08.

Baker, S. M. (2015). Adaptive equilibrium regulation: A balance act in two timescales. Journal of Person-Oriented Research, 1 (1-2), 99-109. DOI: 10.17505/jpor.2015.10.

Bergman, L. R., \& El-Khouri, B. (1987). EXACON: A Fortran 77 program for the exact analysis of single cells in a contingency table. Educational and Psychological measurement, 47(1), 155-161. DOI 10.1177/0013164487471024.

Bergman, L. R., \& Lundh, L-G. (2015). Introduction: The person-oriented approach: Roots and roads to the future. Journal of Person-Oriented Research, 1 (1-2), 1-6. DOI: 10.17505/jpor.2015.01.

Bergman, L. R., \& Magnusson, D. (1997). A person-oriented approach in research on developmental psychopathology. Development and Psychopathology, 9(2), 291- 319. DOI: $10.1017 / \mathrm{S} 095457949700206 \mathrm{X}$.

Bergman, L. R., Magnusson, D., \& El-Khouri, B. M. (2003). Studying individual development in an interindividual context: A person-oriented approach. Vol. 4 in the series Paths through life (D. Magnusson, Ed.). Mahwah, NJ: Erlbaum.

Bergman, L. R., \& Wångby, M. (2014). The person-oriented approach: A short theoretical and practical guide. Eesti Haridusteaduste Ajakiri, 2, 29-49. Doi: 10.12697/eha.2014.21.02b.

Bood SÅ, Archer T, Norlander T (2004) Affective personality in relation to general personality, self-reported stress, coping and optimism. Individ Diff Res 2, 26-37. 
492

493

494

495

496

497

498

499 500

Buhrmester, M. D., Kwang, T., \& Gosling, S. D. (2011). Amazon's mechanical turk: A new source of inexpensive, yet high-quality, data? Perspectives on Psychological Science, 6, $3-5$.

Clark, A., \& Watson, L. A. (1988). Mood and the mundane: relations between daily life events and self-reported mood. Journal of Personality and Social Psychology, 54, 370-376.

Clark, L. A. and Watson, D. (1991). Tripartite model of anxiety and depression: psychometric evidence and taxonomic implications. Journal of Abnormal Psychology, 100, 316-336.

Cloninger, C. R., \& Garcia, D. (2015). The Heritability and Development of Positive Affect and Emotionality. In M. Pluess (Ed.), Genetics of Psychological Well-Being - The Role of Heritability and Genetics in Positive Psychology (97-113). New York: Oxford University Press.

Cloninger, C. R., Svrakic, N. M., \& Svrakic, D. M. (1997). Role of personality self-organization in development of mental order and disorder. Development and Psychopathology, 9, 881906.

Costa, P. T. J., \& McCrae, R. R. (1980). Influence of extroversion and neuroticism on subjective well-being: happy and unhappy people Journal of Personality and Social Psychology, 38, $668-687$.

Crawford, J. R., \& Henry, J. D. (2004). The Positive and Negative Affect Schedule (PANAS): Construct validity, measurement properties and normative data in a large non-clinical sample. British Journal of Clinical Psychology, 43(3), 245.

Diener, E., Colvin, C. R., Pavot, W. G., \& Allman, A. (1991a). The psychic costs of intense positive affect. Journal of Personality and Social Psychology, 61, 492-503. 
514 Diener, E., Sandvik, E., \& Pavot, W. (1991b). Happiness is the frequency, not the intensity, of positive versus negative affect. In F. Strack, M. Argyle, \& N. Schwarz (Eds.), Subjective well-being: An interdisciplinary perspective (pp. 119-139). New York: Pergamon.

Garcia, D. (2011). Adolescents' happiness: the role of the affective temperament model on memory and apprehension of events, subjective well-being and psychological well-being. PhD thesis, University of Gothenburg, Gothenburg, Sweden.

Garcia D (2012) The affective temperaments: differences between adolescents in the big five model and Cloninger's psychobiological model of personality. Journal of Happiness Studies 13, 999-1017. DOI 10.1007/s10902-011-9303-5.

Garcia D \& Archer T (2012) Adolescent life satisfaction and well-being. J Altern Med Res 4, 271-279.

Garcia, D., \& Erlandsson, A. (2011). The Relationship between Personality and Subjective WellBeing: Different Association Patterns when Measuring the Affective Component in Frequency and Intensity. Journal of Happiness Studies, 12, 1023-1034. DOI: $10.1007 / \mathrm{s} 10902-010-9242-6$.

Garcia, D., Kerekes, N., Andersson-Arntén, A-C., \& Archer, T. (2012). Temperament, Character, and Adolescents' Depressive Symptoms: Focusing on Affect. Depression Research and Treatment. DOI:10.1155/2012/925372.

Garcia, D., Rosenberg, P., Erlandsson, A., \& Siddiqui, A. (2010). On Lions and Adolescents: Affective Temperaments and the Influence of Negative Stimuli on Memory. Journal of Happiness Studies, 11, 477-495. DOI: 10.1007/s10902-009-9153-6. 
535 Garcia D, Moradi S (2013) The affective temperaments and well-being: Swedish and Iranian

536

537

538

539

540

541

542

543

544 545

546

547

548

549

550

551

552

553

554

555

556 adolescents' life satisfaction and psychological well-being. Journal of Happiness Studies 14, 689-707. DOI 10.1007/s10902-012-9349-z.

Garcia D, Nima AA, Kjell ONE (2014) The affective profiles, psychological well-being, and harmony: environmental mastery and self-acceptance predict the sense of a harmonious life. PeerJ 2;e259. DOI 10.7717/peerj.259.

Garcia D, Siddiqui A (2009a) Adolecents' affective temperaments: life satisfaction, interpretation and memory of events. The Journal of Positive Psychology 4, 155-167. DOI 10.1080/17439760802399349.

Garcia D, Siddiqui A (2009b) Adolecents' psychological well-being and memory life events influences on life satisfaction with respect to temperamental dispositions. Journal of Happiness Studies 10, 387-503. DOI 10.1007/s10902-008.9096-3.

Goldberg, D. (2011). The heterogeneity of "major depression". World Psychiatry, 10 (3), 226228.

Horton JJ, Rand DG, Zeckhauser RJ. 2011. The online laboratory: conducting experiments in a real labor market. Experimental Economics 4:399-42 DOI 10.1007/s10683-011-9273-9.

Jimmefors, A., Garcia, D., Rosenberg, P., Mousavi, F., Adrianson, L., \& Archer, T. (2014). Locomotion (Empowering) and Assessment (Disempowering) Self-regulatory Dimensions as a Function of Affective Profile in High School Students. International Journal of School and Cognitive Psychology, 2: 103. DOI: 10.4172/1234-3425.1000103.

Keren, G., \& Schul, Y. (2009). Two is not always better than one. A critical evaluation of twosystem theories. Perspectives on Psychological Science, 4, 533-550. 
557 Karlsson E, Archer T (2007) Relationship between personality characteristics and affect: gender and affective personality. Individual Differences Research 5, 44-58.

Kormi-Nouri, R., MacDonald, S., Farahani, M. N., Trost, K., \& Shokri, O. (2015). Academic Stress as A Health Measure and Its Relationship to Patterns of Emotion in Collectivist and Individualist Cultures: Similarities and Differences. International Journal of Higher Education, 4(2), p92. DOI 10.5430/ijhe.v4n2p92.

Laursen, B. (2015). I don't quite get it..: Personal experiences with the person-centered approach. Journal of Person-Oriented Research, 1 (1-2), 42-47. DOI: 10.17505/jpor.2015.05.

Lundh, L-G (2015) The Person as a Focus for Research - The Contributions of Windelband, Stern, Allport, Lamiell, and Magnusson. Journal of Person-Oriented Research, 1 (1-2), 15-33. DOI: 10.17505/jpor.2015.03.

Lux, V., \& Kendler, K. S. (2010). Deconstructing major depression: a validation study of the DSM-IV diagnostic criteria. Psychological Medicine, 40, 1679-16 90.

MacDonald S, Kormi-Nouri R (2013) The affective personality, sleep, and autobiographical memories. The Journal of Positive Psychology: dedicated to furthering research and promoting good practice $8,305-313$.

Molenaar, P. C. M. (2015). On the relation between person-oriented and subject-specific approaches. Journal of Person-Oriented Research, 1 (1-2), 34-41. DOI: 10.17505/jpor.2015.04.

Nima, A. A., Rosenberg, P., Archer, T., \& Garcia, D. (2013). Anxiety, Affect, Self-Esteem, and Stress: Mediation and Moderation Effects on Depression. PLoS ONE 8(9): e73265. DOI:10.1371/journal.pone.0073265. 
580 Norlander T, Bood SÅ, Archer T (2002) Performance during stress: affective personality, age 581 and regularity of physical exercise. Soc Behav Person 30, 495-508.

582 583

584 585 586

Norlander T., von Schedvin H., Archer T. (2005). Thriving as a function of affective personality: relation to personality factors, coping strategies and stress. Anxiety Stress Coping 18, 105-116. 10.1080/10615800500093777.

Palomo T, Beninger RJ, Kostrzewa RM, Archer T (2008) Focusing on symptoms rather than diagnoses in brain functions: conscious and nonconscious expression in impulsiveness and decision making. Neurotoxicity Research 14, 1-20. DOI 10.1007/BF03033572.

Palomo T, Kostrzewa RM, Beninger RJ, Archer T (2007) Treatment consideration and manifest complexity in comorbid neuropsychiatric disorders. Neurotoxicity Research 12, 43-60. DOI 10.1007/BF03033900.

Paolacci G, Chandler J, Ipeirotis PG. 2010. Running experiments on Amazon Mechanical Turk. Judgment and Decision Making 5:411-419.

Rousseeuw, P. J. (1987). Silhouettes: a graphical aid to the interpretation and validation of cluster analysis. Journal of Computational and Applied Mathematics, 20, 53-65.

Santos, J. M., \& Embrechts, M. (2009). On the Use of the Adjusted Rand Index as a Metric for Evaluating Supervised Classification. Artificial Neural Networks, 5769, 175-184. DOI: 10.1007/978-3-642-04277-5_18.

Schimmack, U., \& Diener, E. (1997). Affect intensity: Separating intensity and frequency in repeatedly measured affect. Journal of Personality and Social Psychology, 73, 13131329.

Shapiro DN, Chandler J, Mueller PA. 2013. Using mechanical turk to study clinical populations. Clinical Psychological Science 1:213-220 DOI 10.1177/2167702612469015. 
603 Schütz, E. (2015). The affective profiles model: ill-being and well-being. PhD thesis, University

604

605

606

607

608

609

610

611

612

613

614

615

616

617

618

619

620

621

622

623

624

625 of Gothenburg, Gothenburg, Sweden.

Schütz E, Garcia D, Archer T (2014) Affective state, stress, and type a-personality as a function of gender and affective profiles. International Journal Of Research Studies in Psychology, 3, 51-64. DOI 10.5861/ijrsp.2013.450.

Schütz E, Archer T, Garcia D (2013) Character profiles and adolescents' self-reported affect. Personality and Individual differences, 54, 841-844. DOI 10.1016/j.paid.2012.12.020.

Schütz E, Sailer U, Nima A, Rosenberg P, Andersson-Arntén A.C, Archer T, Garcia D (2013) The affective profiles in the USA: happiness, depression, life satisfaction, and happinessincreasing strategies. PeerJ 1:e156.DOI 10.7717/peerj.156.

Scollon, C. N., Howard, A. H., Caldwell, A. E., and Ito, S. (2009). The role of ideal affect in the experience and memory of emotions. Journal of Happiness Studies, 10, 257-269.

Spector PE, O'Connell BJ (1994) The contribution of personality traits, negative affectivity, locus of control and Type A to the subsequent reports of job stressors and job strains. $\mathrm{J}$ Occup Organ Psychol 67, 1-11.

Tsai, J. L., Knutson, B., and Fung, H. H. (2006). Cultural variation in affect valuation. Journal of Personality and Social Psychology, 90, 288-307.

Tsai, J. L., Miao, F. F., and Seppala, E. (2007). Good feelings in Christianity and Buddhism: religious differ- ences in ideal affect. Personality and Social Psychology Bulletin, 33, $409-421$.

Tsai, J. L., Miao, F. F., Seppala, E., Fung, H. H., and Yeung, D. Y. (2007). Influence and adjustment goals: sources of cultural differences in ideal affect. Journal of Personality and Social Psychology, 92, 1102- 1117. 
626 Valsiner, J. (2015). From person-oriented to person-centered psychology: Abstracting structures

627

628

629

630

631

632

633

634

635

636

637

638

639

640

641

642

643

644

645

646

of relationships. Journal of Person-Oriented Research, 1 (1-2), 7-14. DOI: 10.17505/jpor.2015.02.

Vargha, A., Torma, B., Bergman, L. R. (2015). ROPstat: A general statistical package useful for conducting person-oriented analyses. Journal of Person-Oriented Research, 1, 87-97. DOI: 10.17505/jpor.2015.09.

von Eye, A., \& Bogat, G. A. (2006). Person-oriented and variable-oriented research: Concepts, results, and development. Merrill-Palmer Quarterly, 52(3), 390-420. DOI $10.1353 / \mathrm{mpq} .2006 .0032$

von Eye, A., \& Bogat, G. A., \& Rhodes, J. E. (2006). Variable-oriented and person-oriented perspectives of analysis: The example of alcohol consumption in adolescence. Journal of adolescence, 29, 981-1004. DOI 10.1016/j.adolescence.2006.06.007.

von Eye, A., \& Wiedermann, W. (2015). General linear models for the analysis of single subject data and for the comparison of individuals. Journal of Person-Oriented Research, 1 (1-2), 56-71. DOI: 10.17505/jpor.2015.07.

Watson, D., Clark, L. A. (1994) The PANAS-X Manual for the Positive and Negative Affect Schedule - Expanded form. The University of Iowa Reports Copyright, 1-24.

Watson, D., \& Tellegen, A. (1985). Toward a consensual structure of mood. Psychol Bull 98, $219-235$.

Watson D, Pennebaker JW, Folger R (1986) Beyond negative affectivity: measuring stress and satisfaction in the workplace. J Organ Behav Manag 8, 141-157. 
647 Watson, D., Clark, L. A., \& Tellegen, A. (1988). Development and validation of brief measures 648 of positive and negative affect: The PANAS scale. Journal of Personality and Social 649 Psychology, 54, 1063-1070.

650

651

652

653

654

655

656

657

658

659

660

661

662

663

664

665

666

667

668

669

667 role of negative affectivity. Psychol Rev 96, 234-254.

Wilson KE, Gullone E, Moss S (1998) The youth version of the Positive and Negative affect Schedule. Behav Change 15, 187-193.

4

Watson D, Pennebaker JW (1989) Health complaints, stress and distress: exploring the central

655

656

657

658

659

660

\section{1}

62

\section{3}


670 Legends

671 Table 1. Affective profiles pattern of standardized means for median splits and cluster approaches.

Table 2. Exact cell-wise analysis of two-way frequencies of profiles generated with the median splits and the cluster approaches.

Note: Grey fields in diagonal highlight the cells in which there is a general agreement between approaches when allocating people to specific affective profiles. Black fields highlight the cells in which discrepancies between approaches were found. Rand Index $=.83$.

Type: the observed cell frequency is significantly greater than the expected $(p<.05)$. Antitype: the observed cell frequency is significantly smaller than the expected $(p<.05)$.

- : the observed cell frequency is as expected.

Table 3. Exact cell-wise analysis of two-way frequencies: gender and profiles generated with the median splits and cluster approach, respectively.

Note:

Type (grey fields): the observed cell frequency is significantly greater than the expected $(p<$ $.05)$.

Antitype (black fields): the observed cell frequency is significantly smaller than the expected $(p$ $<.05)$.

- : the observed cell frequency is as expected.

Figure 1. Summary of the main findings during the past 10 years using the affective profiles model by Archer, Garcia, and colleagues.

Figure 2. Distribution of positive and negative affect.

Figure 3ab. Means in positive affect (a: "Joy") and negative affect (b: "Sadness") for each profile derived using the median splits and cluster analysis approaches. 
1

Summary of the main findings during the past 10 years using the affective profiles model by Archer, Garcia, and colleagues 


\section{High Positive Affect}

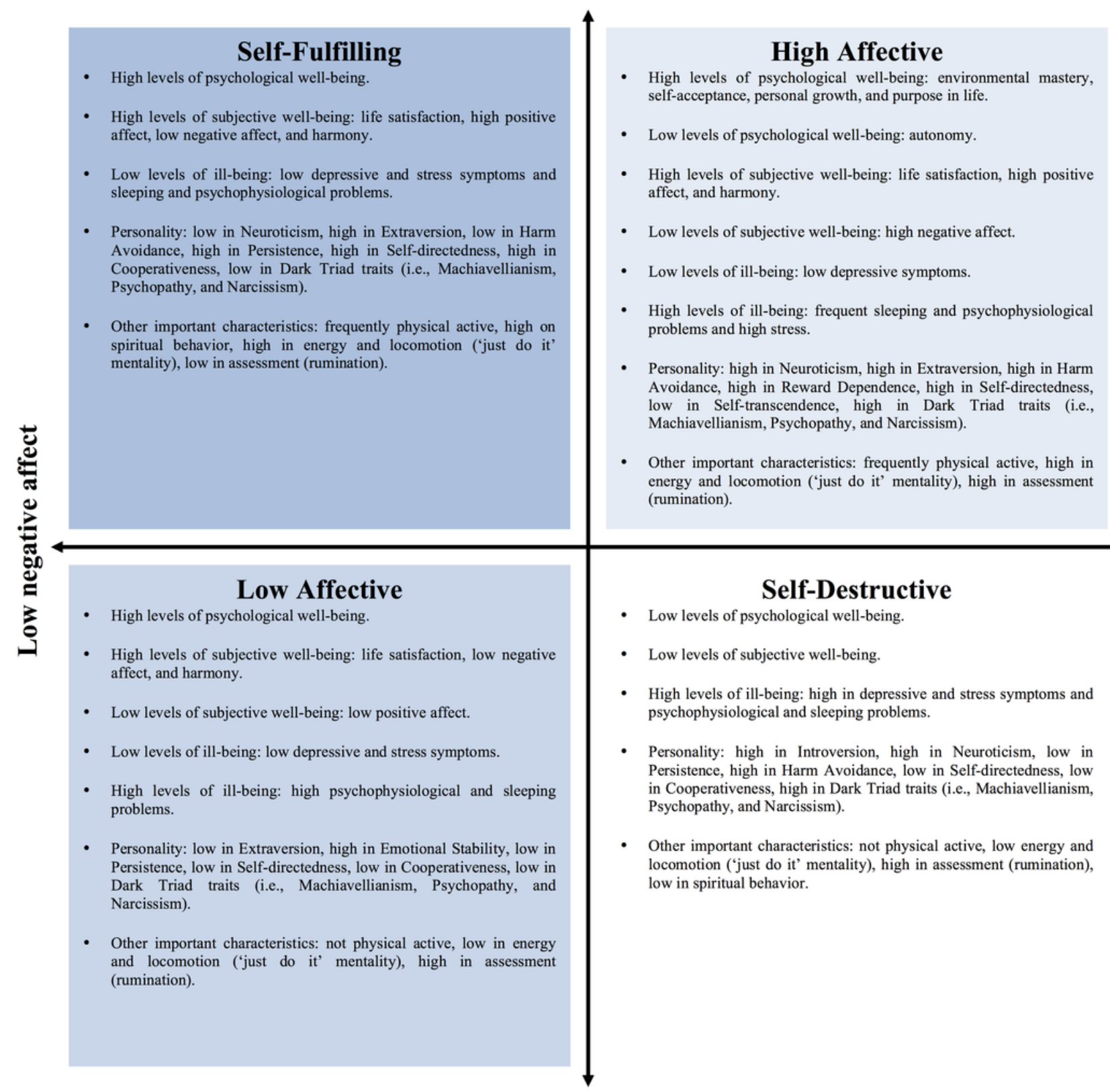

\section{Low positive affect}




\section{Table $\mathbf{1}$ (on next page)}

Affective profiles pattern of standardized means for median splits and cluster approaches

Note: Silhouette coefficient was 0.59 for the median splits method and 0.68 for the cluster

method. Weighted average of cluster homogeneity coefficient was 0.75 for the median splits method and 0.62 for the cluster method. Simple appearance: $0.675<=|z|<=1.000$ (p: 1625\%). ( ): $0.44<=|z|<=0.674$ (p: 25-33\%). $+++: 1.645<=|z|<=2.044$ (p: 2-5\%). 
$1 \quad$ Table 1. Affective profiles pattern of standardized means for median splits and cluster approaches.

\begin{tabular}{|c|c|c|c|c|c|c|c|c|}
\hline & \multicolumn{4}{|c|}{ Median splits } & \multicolumn{4}{|c|}{ Cluster } \\
\hline & Prevalence $(\%)$ & Homogeneity & $\begin{array}{c}\text { Positive } \\
\text { Affect }\end{array}$ & $\begin{array}{c}\text { Negative } \\
\text { Affect }\end{array}$ & Prevalence $(\%)$ & Homogeneity & $\begin{array}{c}\text { Positive } \\
\text { Affect }\end{array}$ & $\begin{array}{c}\text { Negative } \\
\text { Affect }\end{array}$ \\
\hline Low Affective & $441(20)$ & 0.47 & low & low & $640(29)$ & 0.63 & low & (low) \\
\hline High Affective & $529(24)$ & 0.86 & HIGH & (HIGH) & $459(20)$ & 0.53 & . & (HIGH) \\
\hline
\end{tabular}

2 Note: Silhouette coefficient was 0.59 for the median splits method and 0.68 for the cluster method. Weighted average of cluster

3 homogeneity coefficient was 0.75 for the median splits method and 0.62 for the cluster method.

4 Simple appearance: $0.675<=|\mathrm{z}|<=1.000$ (p: $16-25 \%$ ).

( ): $0.44<=|\mathrm{z}|<=0.674$ (p: $25-33 \%$ ).

$6+++: 1.645<=|\mathrm{z}|<=2.044$ (p: $2-5 \%)$.

7

8

9

11

12

13

14

15 
2

Distribution of positive and negative affect

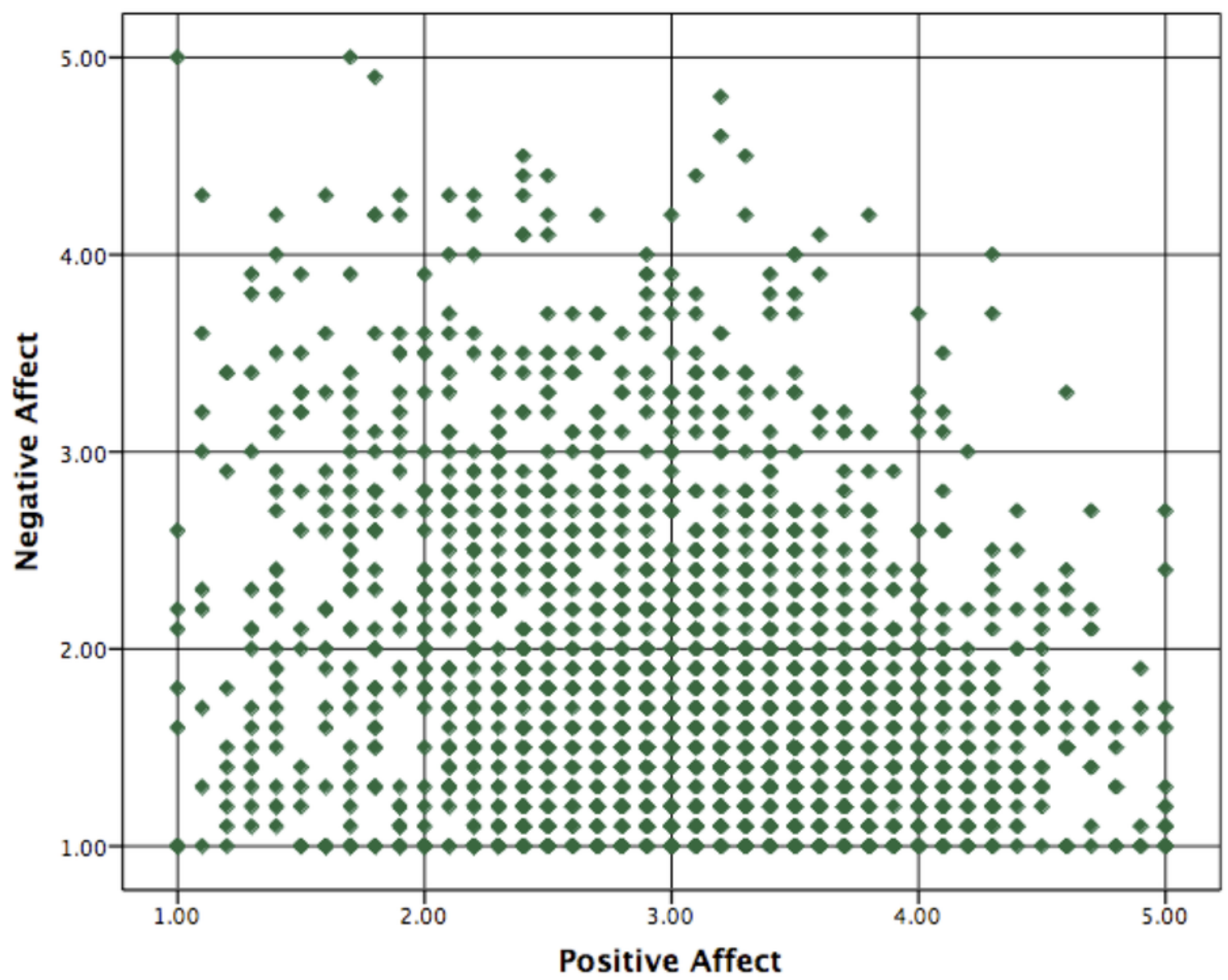


3

Means in positive affect (a: "Joy") and negative affect (b: "Sadness") for each profile derived using the median splits and cluster analysis approaches 
a.

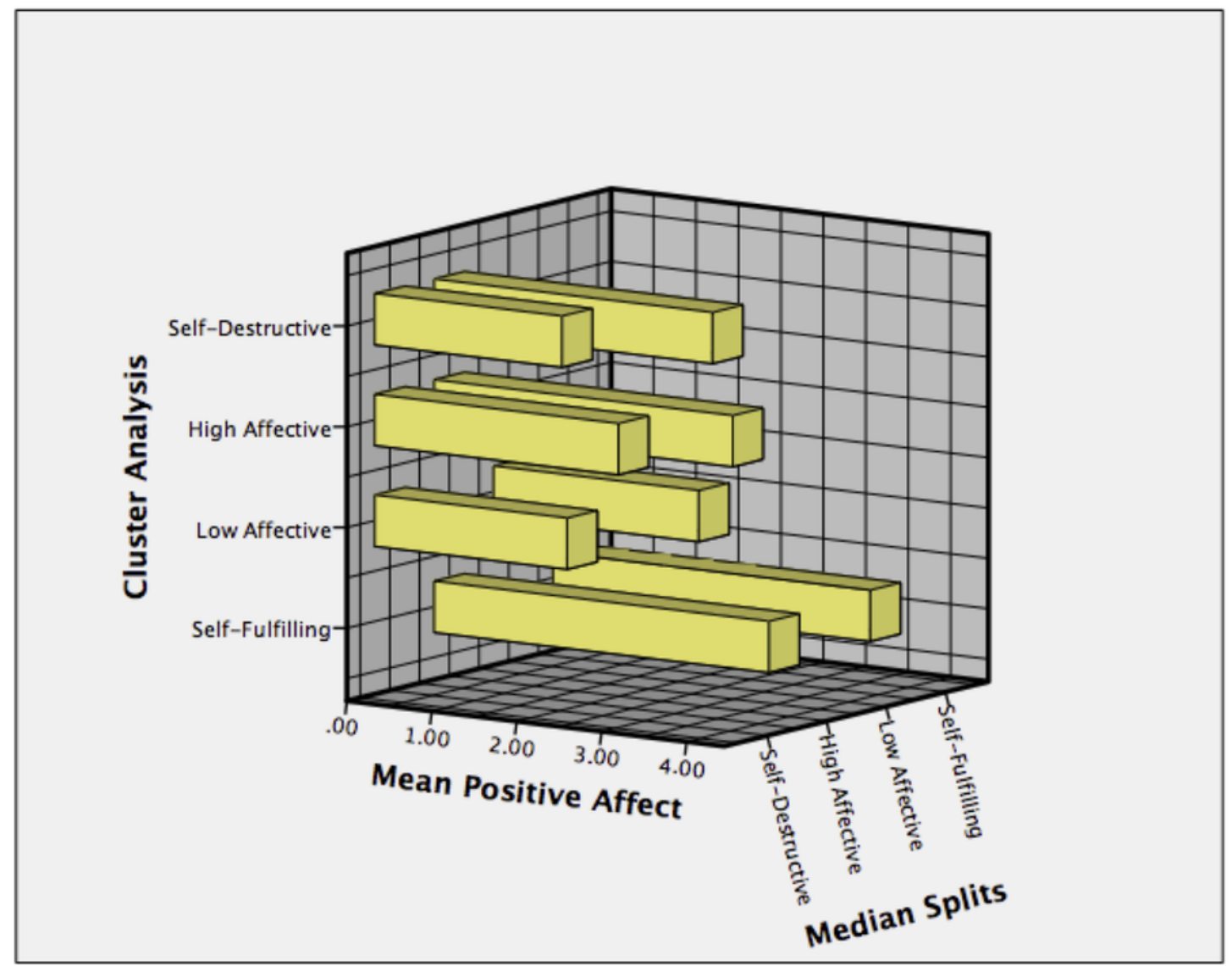

b.

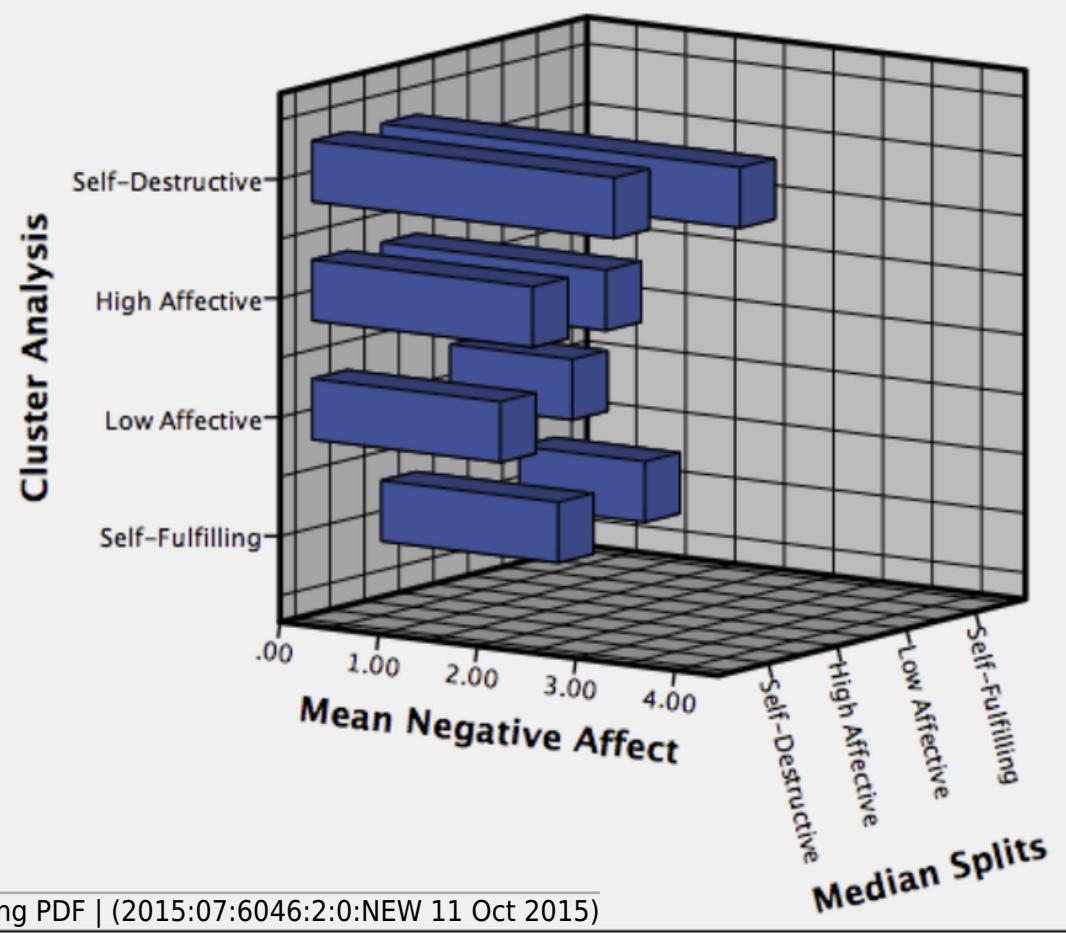




\section{Table 2 (on next page)}

Exact cell-wise analysis of two-way frequencies of profiles generated with the median splits and the cluster approaches

Note: Grey fields in diagonal highlight the cells in which there is a general agreement between approaches when allocating people to specific affective profiles. Black fields highlight the cells in which discrepancies between approaches were found. Rand Index $=.83$. Type: the observed cell frequency is significantly greater than the expected $(p<.05)$. Antitype: the observed cell frequency is significantly smaller than the expected $(p<.05)$. - : the observed cell frequency is as expected. 
Table 2. Exact cell-wise analysis of two-way frequencies of profiles generated with the median splits and the cluster approaches.

\begin{tabular}{|c|c|c|c|c|c|}
\hline & \multicolumn{4}{|c|}{ Cluster analysis } \\
\hline & & Self-fulfilling & Low-Affective & High-Affective & Self-destructive \\
\hline \multirow{12}{*}{ 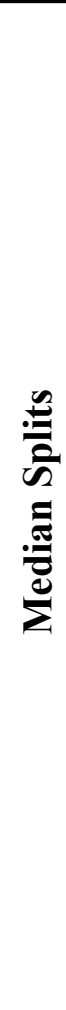 } & Self-fulfilling & Type & Antitype & Antitype & Antitype \\
\hline & Observed & 641 & 0 & 0 & 0 \\
\hline & Expected & 225.00 & 184.00 & 132.23 & 99.40 \\
\hline & Low-Affective & Antitype & Type & Antitype & Antitype \\
\hline & Observed & 0 & 441 & 0 & 0 \\
\hline & Expected & 154.80 & 126.80 & 91.00 & 68.40 \\
\hline & High-Affective & Antitype & Antitype & Type & Antitype \\
\hline & Observed & 140 & 0 & 349 & 40 \\
\hline & Expected & 185.70 & 152.20 & 109.10 & 82.00 \\
\hline & Self-destructive & Antitype & Type & - & Type \\
\hline & Observed & 0 & 199 & 110 & 305 \\
\hline & Expected & 215.52 & 176.60 & 126.70 & 95.20 \\
\hline
\end{tabular}

2 Note: Grey fields in diagonal highlight the cells in which there is a general agreement between approaches when allocating people to specific affective profiles. Black fields highlight the cells in which discrepancies between approaches were found. Rand Index $=.83$.

Type: the observed cell frequency is significantly greater than the expected $(p<.05)$.

5 Antitype: the observed cell frequency is significantly smaller than the expected $(p<.05)$.

6 - : the observed cell frequency is as expected. 


\section{Table 3(on next page)}

Exact cell-wise analysis of two-way frequencies: gender and profiles generated with the median splits and cluster approach, respectively

Note: Type (grey fields): the observed cell frequency is significantly greater than the expected $(p<.05)$. Antitype (black fields): the observed cell frequency is significantly smaller than the expected $(p<.05)$. - : the observed cell frequency is as expected. 
1 Table 3. Exact cell-wise analysis of two-way frequencies: gender and profiles generated with the median splits and cluster approach, 2 respectively.

\begin{tabular}{|c|c|c|c|c|}
\hline \multirow{2}{*}{ Gender } & \multicolumn{4}{|c|}{ Median splits affective profiles } \\
\hline & Self-fulfilling & Low-Affective & High-Affective & Self-destructive \\
\hline $\begin{array}{c}\text { Male } \\
\text { Observed }(\%) \\
\text { Expected }\end{array}$ & $\begin{array}{c}- \\
351(54.80 \%) \\
334.20\end{array}$ & $\begin{array}{c}- \\
235(53.30 \%) \\
229.90\end{array}$ & $\begin{array}{c}- \\
283(53.50 \%) \\
275.80\end{array}$ & $\begin{array}{c}\text { Antitype } \\
291(47.40 \%) \\
320.10\end{array}$ \\
\hline $\begin{array}{c}\text { Female } \\
\text { Observed }(\%) \\
\text { Expected }\end{array}$ & $\begin{array}{c}- \\
290(45.20 \%) \\
306.80\end{array}$ & $\begin{array}{c}- \\
206(46.70 \%) \\
211.10\end{array}$ & $\begin{array}{c}- \\
246(46.50 \%) \\
253.20\end{array}$ & $\begin{array}{c}\text { Type } \\
323(52.60 \%) \\
293.90\end{array}$ \\
\hline Expected & \multicolumn{4}{|c|}{ Cluster analysis affective profiles } \\
\hline $\begin{array}{c}\text { Male } \\
\text { Observed (\%) } \\
\text { Expected }\end{array}$ & $\begin{array}{c}\text { Type } \\
431(55.20 \%) \\
407.20\end{array}$ & $\begin{array}{c}- \\
336(52.50 \%) \\
333.70\end{array}$ & $\begin{array}{c}- \\
251(54.70 \%) \\
239.30\end{array}$ & $\begin{array}{c}\text { Antitype } \\
291(41.20 \%) \\
320.10\end{array}$ \\
\hline $\begin{array}{c}\text { Female } \\
\text { Observed }(\%) \\
\text { Expected }\end{array}$ & $\begin{array}{c}\text { Antitype } \\
350(44.80 \%) \\
373.80\end{array}$ & $\begin{array}{c}- \\
304(47.50 \%) \\
306.30\end{array}$ & $\begin{array}{c}- \\
208(45.30 \%) \\
219.70\end{array}$ & $\begin{array}{c}\text { Type } \\
203(58.80 \%) \\
165.10\end{array}$ \\
\hline
\end{tabular}

3 Note:

4 Type (grey fields): the observed cell frequency is significantly greater than the expected $(\mathrm{p}<.05)$.

5 Antitype (black fields): the observed cell frequency is significantly smaller than the expected $(\mathrm{p}<.05)$.

$6-$ : the observed cell frequency is as expected. 